\title{
Using artificial neural networks and strain gauges for the determination of static loads on a thin square fully-constrained composite marine panel subjected to a large central displacement
}

\begin{abstract}
Current methods of estimating the behaviour of marine composite structures under pressure due to slamming as a result of high waves are based on trial and error or oversimplification. Normally under these conditions the non-linearities of these structures are often neglected and, in order to compensate, an overestimated safety factor is employed. These conservative approaches can result in heavier and overdesigned structures. In this paper, a new semi-empirical method is proposed that overcomes some of
\end{abstract}

Mohammad Reza Ramazani received his MEng degree in mechanical engineering from the University of Birmingham in 2009. He is currently studying for a PhD jointly funded by Bournemouth University and BAE Surface Ships Ltd to investigate in-service load monitoring of marine structures. His interests include artificial intelligence and computer-aided engineering.

Professor Siamak Noroozi received his PhD from Sheffield University in 1986 in the area of finite element analysis coupled with boundary element analysis. He currently holds the Chair in Advanced Technology at Bournemouth University. His research interests are finite element analysis, boundary element analysis, biomechanics, condition monitoring, general stress analysis, photoelasticity, alternative numerical analysis, composite technology and aeroelasticity.

Dr Philip Sewell received his BEng degree in mechanical engineering from the University of the West of England in 1999 and a PhD in the field of prosthetic design in 2003. He is currently employed as a Senior Academic in Design Simulation at Bournemouth University. His research interests include the design of novel tools for prosthetic fitting, the development of techniques to determine prosthetic interfacial pressure distributions and experimental and numerical stress analysis.

Rasoul Khandan received his BSc degree in mechanical engineering from the Isfahan University of Technology in 2004 and an MSc in mechanical engineering (applied design) in 2008 from Shiraz University. He is currently working on modelling, simulation and optimisation of composite materials as a PhD researcher in the Design Simulation Research Centre at Bournemouth University. His main research interest is modelling and the application of composite and smart materials.

Professor Bob Cripps received his degree in ship science from the University of Southampton in 1976. He was awarded an Honorary Doctor of Engineering from Bournemouth University in 2005. He is currently a Director of Longitude Consulting Engineering, part of London Offshore Consultants (LOC). He is a Visiting Professor at Bournemouth University and a Royal Academy of Engineering Visiting Professor in the Principles of Engineering Design at the University of Southampton.

Mohammad Reza Ramazani*, Siamak Noroozi, Philip Sewell and Rasoul Khandan are with the School of Design, Engineering \& Computing, Bournemouth University, Bournemouth, UK.

Bob Cripps is with Longitude Consulting Engineers Ltd, Southampton, UK.

*Corresponding author. Tel: 01202 961528; Email: mramazani@ bournemouth.ac.uk these problems. This work involved the use of an artificial neural network (ANN) combined with strain gauge data to enable real-time in-service load monitoring of large marine structural panels. Such a tool has other important applications, such as monitoring slamming or other transient hydrostatic loads that can ultimately affect fatigue life. To develop this system, a glass fibre-reinforced polymer (GFRP) composite panel was used due to its potential for providing a non-linear response to pressure or slamming loads. It was found that the ANN was able to predict normal loads applied at different locations on the panel accurately. This method is also capable of predicting loads on the marine structure in real time.

Keywords: Composite, marine, artificial intelligence, artificial neural network, structural analysis, load, non-linear structures, large displacement analysis.

\section{Introduction}

In addition to hydrostatic and mass-related loads that can be evaluated with a high degree of accuracy and confidence, it is also desirable to measure transient loads due to slamming as a result of high waves. The current practice to determine wave loads is based on applying standard rules, which often relies on conservative methods due to large uncertainties in the theoretical treatment used for wave load predictions. This leads to a craft that is heavier and slower than it could otherwise be.

Although sea has an irregular and arbitrary condition, the overall condition can be predicted statistically by superimposing a series of different regular waves of varying heights, lengths, directions and phase ${ }^{[1-3]}$. In order to define the sea-state that the craft are expected to encounter during their lifetime, an enormous amount of data regarding ocean waves has been collected. Hogben et a $l^{[4]}$ collected comprehensive data regarding ocean waves from 104 ocean areas covering all major shipping routes. Having more information about sea states, the wave-induced loads on the craft structure and the response to such loads may be estimated.

Techniques used to measure hydrodynamic loads use nonlinear equations due to the random and irregular nature of the sea, resulting in a very expensive and time-consuming analysis. Methods have been developed in order to simplify such an analysis ${ }^{[5]}$. Strip theory is one of the most well-known techniques used to determine the wave-induced loads ${ }^{[6,7]}$. The principle is that the craft's hull is divided into a number of segments or strips. The forces acting on the hull are then calculated separately on each segment using a two-dimensional flow theory. This method ignores the longitudinal component of relative velocity and any type of interaction between the different segments. Other shortcomings of this theory include ignoring three-dimensional or viscous effects 
as well as the inability to account for the above-water hull form. In order to resolve the problem with compatibility between strips, flexible beam strip theories were developed that account for the bending and shear stiffness of the hull ${ }^{[8]}$. Although this kind of theory can estimate the distortional higher frequency responses of a hull to slamming and lashing excitation, it is still linear analysis and extreme response is not well modelled.

The accuracy of the strip theory and other codes has been investigated by several researchers and the error associated with predicting a mid-ship bending moment using strip theory is of the order of $10 \%$ to $20 \%$. This accuracy is reduced further towards the ends of the vessel and as seas become progressively more beam-on ${ }^{[9]}$. Clarke ${ }^{[10]}$ conducted many on-board measurements employing several Royal Navy (RN) ships. The results indicated that strip theory over-estimates wave bending moments, particularly at larger wave heights. Furthermore, the hogging bending moment was over-predicted more so than the sagging moment. It is concluded that these techniques are only accurate for moderate sea conditions and ship speeds meaning an extreme load causing a large displacement in panels is impossible to measure. Moreover, doubts also exist in many of the assumptions that involve stochastic/random data or procedures involving environmental and operational conditions. This is due to the fact that sometimes environmental and operational conditions are difficult to define accurately in advance and therefore assumptions are needed ${ }^{[11]}$.

In order to improve the accuracy of estimation, especially in short waves, many numerical methods considering the threedimensional effects have been introduced. Among them are the three-dimensional Green function method ${ }^{[12]}$ and Rankine source method $^{[13-15]}$ based on three-dimensional potential theory. The benefits of these methods include taking the three-dimensional effects into account, having good stability of computations and a moderate computing time. Hence, they are considered as suitable design tools replacing the strip methods.

A review by Phelps ${ }^{[9]}$ indicates that non-linear theories and three-dimensional load prediction methods have been introduced, but these require greater computational effort and have not yet proven to be significantly more accurate than the two-dimensional methods. It is concluded that a novel technique is required to overcome current limitations in the practices used to measure and estimate loads experienced by the hull of a small, high-speed boat operating in a seaway. Furthermore, as vessels and craft are, in most cases, extremely complicated structures, the mechanical properties, or relationship between externally-induced excitation and structural responses, are difficult to formulate. An appropriate load monitoring system and technique has to be developed for naval assets and large structures ${ }^{[16]}$.

A novel approach for the determination of pressure loads experienced by marine structures is the utilisation of artificial neural networks (ANN) as an inverse method. In a study by Cao et $a l^{[16]}$, an approach was developed to identify the loads acting on aircraft wings, where an ANN was utilised to model the load-strain relationship for structural analysis. The research demonstrated that using an ANN to identify loads is feasible and a well-trained ANN reveals an extremely fast convergence and a high degree of accuracy in the process of load identification for a cantilevered beam model. In a study by Amali et $a l^{[17]}$, it is illustrated that ANN can be combined with experimental methods to create a hybrid inverse problem analysis tool or inverse problem engine. The hybrid approach can be applied to both direct problems (calculation of the structural response from known loads applied to the structure) and inverse problems (calculation of the applied load from a known structural response). Additionally, the approach avoids the need to have information on the component geometry and material properties ${ }^{[18,19]}$.

Ramazani et $a l^{[20]}$ have recently shown that the inverse problem approach can be used to estimate low loads applied on a composite marine panel from a small deflection and its associated strain measurements. A comparison of the ANN loads with the actual applied loads indicated a very good performance of the methodology. This was achieved in real time, providing an accurate load history for a component without requiring knowledge of the material properties or component geometry. However, a large load results in a large displacement in the panel, where the displacement is no longer predictable. This implies that the superposition method of generating training data for a small displacement can no longer be applied here. However, marine structures do experience large displacement and for that reason load prediction is essential. This paper reports on the research undertaken to further develop the ANN methodology to quantify static pressure/central load on a composite marine panel from its non-linear structural response.

\section{Methodology}

The methodology employed to evaluate the suitability of an ANN as an inverse problem is presented in this section. A backpropagation ANN was designed, developed and trained within the Matlab simulation environment (Mathworks, Natick, Massachusetts, USA) to measure transverse load on a flat composite marine panel. The estimated output was then validated by comparing it against both experimental and numerical data.

\subsection{Inverse problem analysis methodology}

Inverse problem analysis is based on accurately calculating the external loads or boundary conditions that generate a known strain at pre-determined locations on a structure. An ANN, as an inverse problem solver, can be utilised to determine a relationship between the cause and its effect ${ }^{[20]}$. In this study, the static loads (the cause/ output) on a composite panel are quantified by acquiring repeatable strain responses (the effect/input) to these loads from the panel. Introducing these examples to an ANN, the system can learn and form the relationships between the input (strains) and output (load) through the transfer function. The ANN requires a number of known input and output data for training (ie relating the ANN inputs to outputs using a transfer function and series of weighting values). Once the ANN is sufficiently trained it can be utilised to estimate the output in real time. New inputs (problem data) can then be presented and the load can be estimated in real time.

\subsection{Experimental set-up}

The structure under consideration was a $1 \mathrm{~m}^{2}$ glass fibre-reinforced fibre polymer/plastic (GFRP) marine composite panel (Figure 1). The sample GFRP composite panel used was made of seven layers of stitched biaxial \pm 45 E-glass cloth with Ampreg 22 epoxy resin system, hand laid-up with a total thickness of $5 \times 10^{-3} \mathrm{~m}$. The fibres were aligned parallel to the edges of the panel. Table 1 shows the experimental mechanical properties of the glass fibre as provided by the manufacturer.

The panel was divided into a four-by-four grid producing sixteen equal regions, each with an area of $0.25 \times 0.25 \mathrm{~m}^{2}$ (Figure

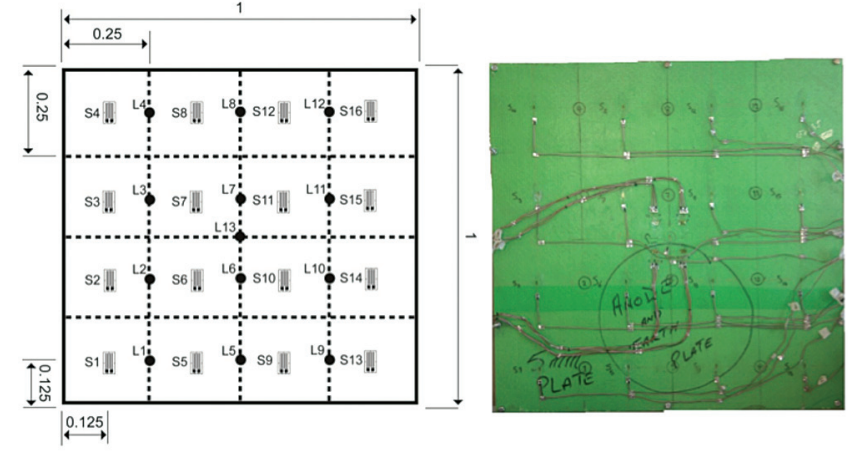

Figure 1. Schematic of composite panel indicating strain gauge and loading locations 
Table 1. Panel material specification provided by SP Gurit Systems (Newport, Isle of Wight, UK)

\begin{tabular}{|c|c|c|c|c|c|}
\hline \multicolumn{3}{|l|}{ Material name } & \multicolumn{3}{|c|}{ XE905 } \\
\hline \multicolumn{3}{|l|}{ Material type } & \multicolumn{3}{|c|}{ Stitched biaxial } \\
\hline \multicolumn{3}{|l|}{ Fibre volume fraction } & \multicolumn{3}{|l|}{0.46} \\
\hline Longitudinal property & \multicolumn{2}{|l|}{ Units } & \multicolumn{3}{|c|}{ Units } \\
\hline Longitudinal tensile modulus & $\mathrm{N} / \mathrm{mm}^{2}$ & 21220 & Poisson's ratio (longitudinal strain) & & 0.120 \\
\hline Longitudinal tensile strength & $\mathrm{N} / \mathrm{mm}^{2}$ & 318.3 & Poisson's ratio (transverse strain) & & 0.120 \\
\hline Longitudinal compressive modulus & $\mathrm{N} / \mathrm{mm}^{2}$ & 21220 & Longitudinal coeff. of thermal expansion & $10-6 /{ }^{\circ} \mathrm{K}$ & 14.62 \\
\hline Longitudinal compressive strength & $\mathrm{N} / \mathrm{mm}^{2}$ & 254.6 & Transverse coeff. of thermal expansion & $10-6 /{ }^{\circ} \mathrm{K}$ & 14.62 \\
\hline \multicolumn{3}{|l|}{ Transverse property } & Density & $\mathrm{kg} / \mathrm{m}^{3}$ & 1786 \\
\hline Transverse tensile modulus & $\mathrm{N} / \mathrm{mm}^{2}$ & 21220 & Structural ply thickness & $\mathrm{mm}$ & 0.75 \\
\hline Transverse tensile strength & $\mathrm{N} / \mathrm{mm}^{2}$ & 318.3 & Actual ply weight & $\mathrm{g} / \mathrm{m}^{2}$ & 1364 \\
\hline Transverse compressive modulus & $\mathrm{N} / \mathrm{mm}^{2}$ & 21220 & Shear thickness & $\mathrm{mm}$ & 0.75 \\
\hline Transverse compressive strength & $\mathrm{N} / \mathrm{mm}^{2}$ & 254.6 & & & \\
\hline \multicolumn{3}{|l|}{ Shear properties } & \multicolumn{3}{|l|}{ Derived shear properties @ $\pm 45^{\circ}$} \\
\hline Interlaminar shear modulus & $\mathrm{N} / \mathrm{mm}^{2}$ & 3050 & Shear material name & \multicolumn{2}{|c|}{1 x XE905@ @ $\pm 45^{\circ}$} \\
\hline Interlaminar shear strength & $\mathrm{N} / \mathrm{mm}^{2}$ & 36.6 & Axial modulus with fibres @ $\pm 45^{\circ}$ & $\mathrm{N} / \mathrm{mm}^{2}$ & 9737 \\
\hline In-plane shear modulus & $\mathrm{N} / \mathrm{mm}^{2}$ & 3050 & Shear modulus with fibres @ $45^{\circ}$ & $\mathrm{N} / \mathrm{mm}^{2}$ & 9471 \\
\hline In-plane shear strength & $\mathrm{N} / \mathrm{mm}^{2}$ & 46.1 & Poisson's ratio with fibres $@ \pm 45^{\circ}$ & & 0.596 \\
\hline
\end{tabular}

1). The bottom surface of the panel was supported on all four edges using aluminium bars, each $0.0381 \mathrm{~m}$ high, $0.01905 \mathrm{~m}$ wide and $1 \mathrm{~m}$ long. Sixteen linear electrical resistance strain gauges (ERSG) (S1-16) were bonded to the centre of each region (specification in Table 2). Two eight-channel NI cDAQ 9236 modules mounted on NI cDAQ 9174 chassis (National Instruments Corporation, Austin, Texas, USA) were used as the strain monitoring and control data acquisition system with a resolution of $+/-0.1$ microstrain. The system provides differential inputs to monitor sixteen strain gauges at up to 10,000 samples per second. The strain data was collected using Matlab, utilising Matlab Data Acquisition Toolbox capabilities.

\section{Table 2. Strain gauge specification}

\begin{tabular}{|l|c|}
\hline Type & General purpose linear gauge \\
\hline Resistance & $350 \mathrm{ohms} \pm 0.6 \%$ \\
\hline Gauge factor & $2.100 \pm 0.5 \%$ \\
\hline Gauge length & $6.35 \mathrm{~mm}$ \\
\hline Gauge width & $2.54 \mathrm{~mm}$ \\
\hline
\end{tabular}

Normal loads were randomly applied to the top surface of the panel at thirteen grid intersections (L1-13). Depending on the proximity of the gauge to the applied loads, different gauges exhibited different levels of sensitivity, which was as expected. To produce efficient training data the strain data should be captured at the sensitive regions (ie the strain at those locations must vary significantly due to changes in load level). In addition, the strain data collected must provide a unique response for each load distribution. If strain is collected from non-sensitive regions of the panel and/or the strain data collected is not unique for each load distribution the ANN is less likely to be able to find a function relating the input and output.

\subsection{Generation of training data}

Many small marine craft hulls are manufactured from fibreglass strengthened by wood or foam. Their characteristics are such that their thickness is small compared to their other dimensions. In this study, a GFRP panel has been utilised to represent a section of the hull. Panels can be classified according to their thickness and their lateral deflection compared to their thickness ${ }^{[21]}$. They can be classified as: (1) thick plate, small deflection; (2) thin plate and small deflection; (3) thin plate, large deflection; or (4) very thin plate (membranes) with either small deflection or large deflection. In all cases the solutions are approximate, not exact or closed form. The deflection at the centre of a plate subject to pressure is offered by Westergaard and Slater ${ }^{[22]}$ and is based on the modified flexure theory of plates where, depending on the plate aspect ratio, edge boundary conditions and load, different approximate empirical solutions are found. In such cases, a small displacement is defined as displacement less than or equal to half the thickness of the plate. If the displacement exceeds this limit then the problem is treated as a non-linear problem where the displacement can no longer be accurately predicted using the above theory. This is due to highly non-linear double curvature deformation, unlike the displacement function stated above. In large displacement analysis, the transverse shear can also no longer be ignored and if the panel is composite then the transverse shear requires further special treatment. In such cases, the classical inverse approach used previously, based on utilising data generated from superposition, can no longer be employed due to the complexity of the displacement function.

For non-linear structures an alternative approach is needed in order to generate the required training data. There are two ways in which such data can be generated: (a) experimentally; or (b) using a non-linear finite element analysis (FEA) solver. Generating the required training data experimentally is very time consuming and labour intensive. Therefore, non-linear FEA analysis using a script that allowed automatic generation of a random load on the panel was utilised to generate the training data. Abaqus 6.10-1 FEA software (Dassault Systèmes Simulia Corp, Rhode Island, USA) was used. A script function written in Python language was used to iteratively run the software in a batch using different random loads applied at each of the thirteen loading locations on the panel. The FEA model was initially validated to ensure that it represented the actual panel accurately. The validation was achieved by comparing strains collected experimentally with the FEA strains under the same loading conditions. Loads from $100 \mathrm{~N}$ to $800 \mathrm{~N}$ applied in 100 $\mathrm{N}$ increments were placed on the panel one at a time at locations $\mathrm{L} 1$ to $\mathrm{L} 13$. The strain readings at locations $\mathrm{S} 1$ to $\mathrm{S} 16$ on the panel were saved for each test. The same tests were performed with FEA to compare with the experimental results.

Once validated, a large number of training (load/strain 
response) data was able to be generated from the FEA model. In order to increase the efficiency of generating the training data, it was possible to reduce the number of FEA models required to establish the non-linear strain response for each gauge location. This was achieved by fitting non-linear curves to data collected for each strain location and using the curves to interpolate strain data for different load magnitudes.

The structural responses of the panel in terms of strain were saved to be used as the input training dataset. The corresponding load for each input dataset was also saved and utilised as the output training set. Some of these input and outputs were saved separately for testing the network and error minimisation. In this study, sixteen single strain gauge readings (inputs) and thirteen applied loads (outputs) constitute one training dataset. At each loading location (L1-L13), a static load ranging between $24.525 \mathrm{~N}$ and $784.8 \mathrm{~N}$ was applied in steps of $24.525 \mathrm{~N}$. In total, 1040 training datasets were generated from the non-linear FEA model.

\subsection{ANN architecture/topology}

ANN analysis often requires a high number of individual loops to determine the best solution. However, the training time can be reduced (ie reduce the number of loops to minimise the error equation) by pre-processing the data that is given to the network to train. Having multiple hidden layers of neurons with non-linear transfer functions (such as tan-sig and log-sig) enables the network to understand both non-linear and linear relationships between input and output data. Unsatisfactory performance of the ANN can be due to a wide range of reasons, such as:

an unsuitable ANN architecture or learning method;

$\square$ insufficient representative data (not enough example strain/load data);

$\square$ inadequate pre-processing (noisy data from data acquisition system ignored);

$\square$ unsuitable ANN training parameters.

Most of the time this is not the case and the ANN will be well trained and perform satisfactorily, even on a new untrained dataset. Key architectural issues that can be optimised include: (i) the number of layers in the ANN; (ii) the number of neurons per layer; (iii) the type and parameters of the neuron, which are usually the same throughout; and (iv) the number of calculations per iteration during learning and recall.

The Matlab Artificial Neural Network toolbox was used in this study to generate two different backpropagation ANN architectures in order to compare their performance. The architectures utilised were:

$\square$ One network with sixteen neurons in the input layer and thirteen neurons in the output layer is trained to estimate the load on the panel from the strain responses (Figure 3).

$\square$ Thirteen networks each with sixteen neurons in the input layer and one neuron in the output layer are trained and used to estimate the load on the panel from the strain responses (Figure 4). strain reading as an input is introduced to the network, it will be able to estimate the loads that caused those structure responses. Depending on how well the network is trained (the performance of the network), there will be an error between the output dataset and the network estimated output (load).

\subsection{ANN validation and performance}

The validity and performance of the ANN method was evaluated by comparing the load estimated by the ANN with known loads applied to the panel, which were not seen by the network during the training process. The first validation study utilised load and strain data generated from the FEA model and was compared with estimated loads from the ANN. In the second study, problem strain data was captured directly from the panel and again the estimated loads were compared with the actual applied loads.

\section{Results}

The validity of utilising FEA for training data generation and the ANN validity and performance are detailed in the following sections.

\subsection{FEA model validation}

Figure 2 indicates that for loading only location L13, there is reasonable agreement between the strain readings (S6 and S10) of FEA tests and experimental tests. The average percentage error is less than $7 \%$. These results indicate that the FEA model can be used confidently to simulate various loading conditions and to generate the required training input data.
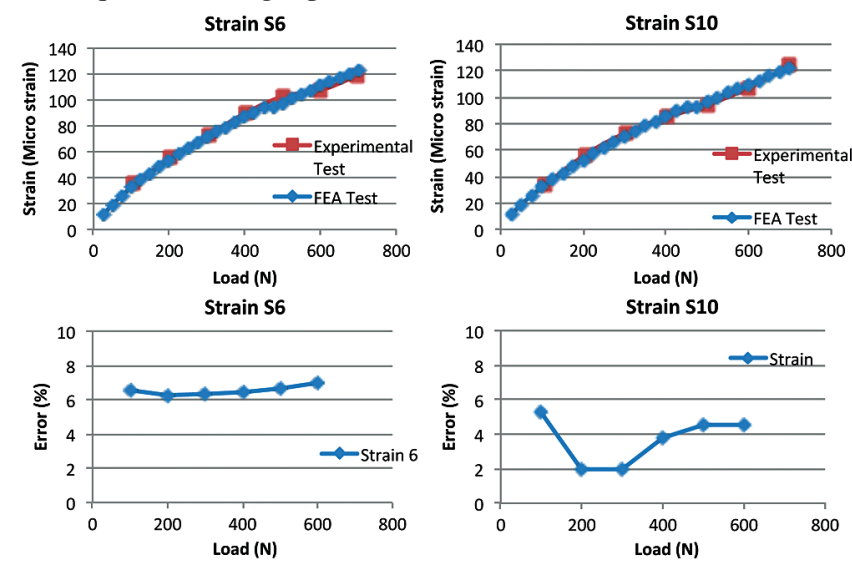

Figure 2. Comparison of FEA and experimental data of selected strain gauges

\subsection{ANN validation and performance}

As mentioned in Section 2.4, two different methods are employed to define the networks. Table 3 lists the major parameters of the network architecture used in the two methods. It was determined,
The number of hidden layers and neurons in each hidden layer of the two network architectures were flexible. These were dependent on the complexity of the training datasets and were optimised according to the network performance. The sum of squared errors (SSE) and mean of squared errors (MSE) are common network performance indicators. Through the testing of various network architectures, the optimum network having the lowest performance indicator can be determined. Once the ANN is trained, it can be employed to estimate new loading cases where the same patterns exist. In other words, whenever the same pattern of

\section{Table 3. ANN architectures}

\begin{tabular}{|l|c|c|}
\hline & $\begin{array}{c}1 \text { network with 16 strain inputs } \\
\text { and 13 load outputs }\end{array}$ & $\begin{array}{c}\text { 13 networks each with 16 } \\
\text { strain inputs and 1 load output }\end{array}$ \\
\hline Number of networks & 1 & 13 \\
\hline Architecture & \multicolumn{2}{|c|}{ Feed forward backpropagation } \\
\hline Number of layers in each network & 2 & 1 \\
\hline Range of load estimation & $24.525-784.8(\mathrm{~N})$ & $24.525-784.8(\mathrm{~N})$ \\
\hline No of inputs (surface strains) & 16 & 16 \\
\hline No of output layer neurons (loads) & 13 & 1 \\
\hline No of each hidden layer of neurons & {$[2020]$} & 1040 \\
\hline Number of training patterns & 1040 & 1040 \\
\hline Number of testing patterns & 1040 & \\
\hline
\end{tabular}


through the testing of various network architectures, that the optimum network (lowest SSE) for method one had two hidden layers with twenty neurons and used a tan-sig transfer (Figure 3). The output layer had thirteen neurons (representing the thirteen loads to be estimated) and used a pure-lin transfer function. Similarly, it was determined that the thirteen networks for method two had one hidden layer each with fifty neurons and used a tansig transfer (Figure 4). The output layer of each network had one neuron (each network estimates the corresponding load of one location) and used a pure-lin transfer function.

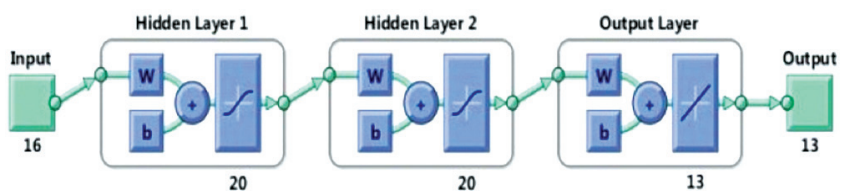

Figure 3. Matlab representation of ANN architecture 1

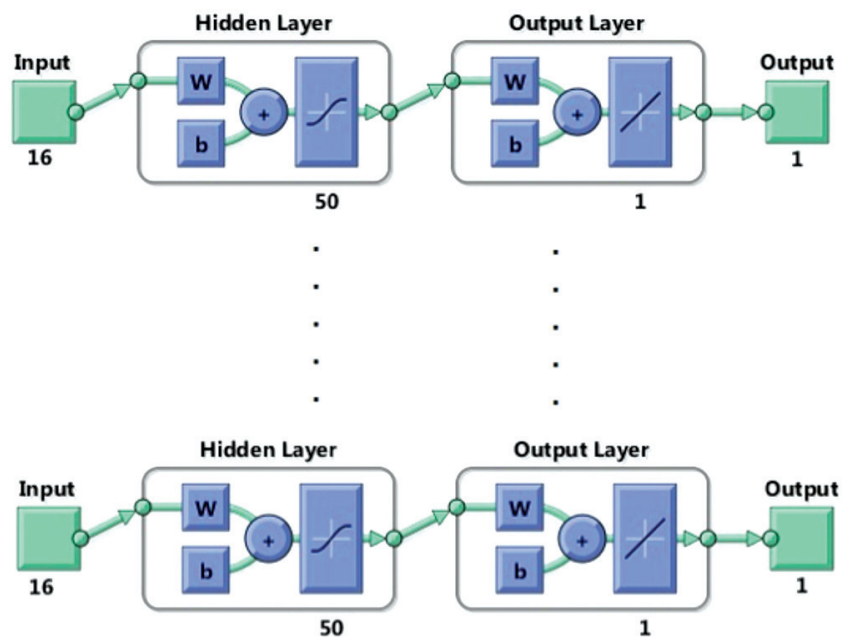

Figure 4. Matlab representation of ANN architecture 2

In this study, SSE is used as a performance indicator. Once the networks were trained, SSE values between the estimated loads and training load data were calculated. Each network has an individual SSE value. This means that although the first method has only one SSE value, the second method had thirteen SSE values. Figure 5 indicates the SSE performance of all thirteen networks, each having sixteen inputs (all strain readings) and one output (load at one location) generated from the second network architecture.

In order to compare the two methods, the summation of all the networks' SSE values in the second method is compared to the SSE value of the first method, when only a network with sixteen inputs and thirteen outputs were used to train the system. As it is indicated in Figure 6, a better performance for the second method is achieved.

In addition to having a better performance, the second method has more flexibility. This means having thirteen independent networks; for each load location a separate new network architecture and parameters can be employed. For instance, the sum of the estimation performances of networks in the second method can be improved by changing the network architecture of those networks (eight and twelve from Figure 5) having relatively higher SSE values. As it is illustrated in Table 4 , for locations eight and twelve, networks with two layers with twenty neurons are used. The improvement in SSE for networks eight and twelve with the new architectures can be seen in Figure 7.

In order to investigate the capability of the ANN to estimate loads in real time, once the ANN is trained new strain data from different

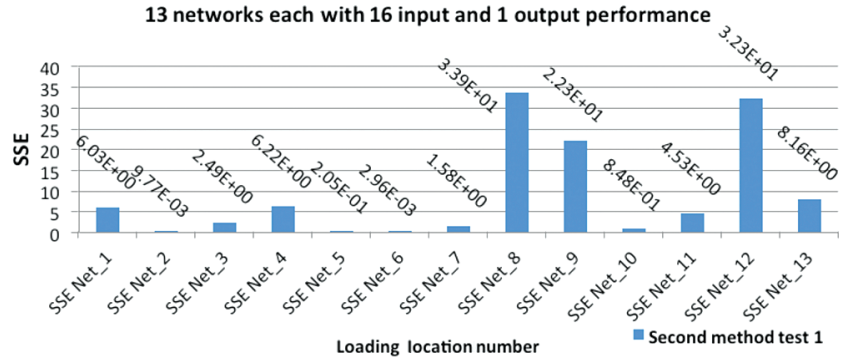

Figure 5. SSE performance of network architecture 2

\section{Sum of squared error comparison}

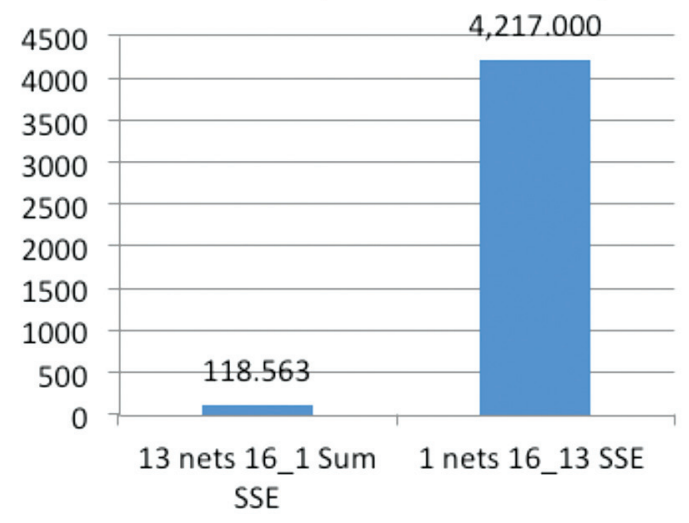

Test 1

Figure 6. Comparison of the SSE values of the two network architectures

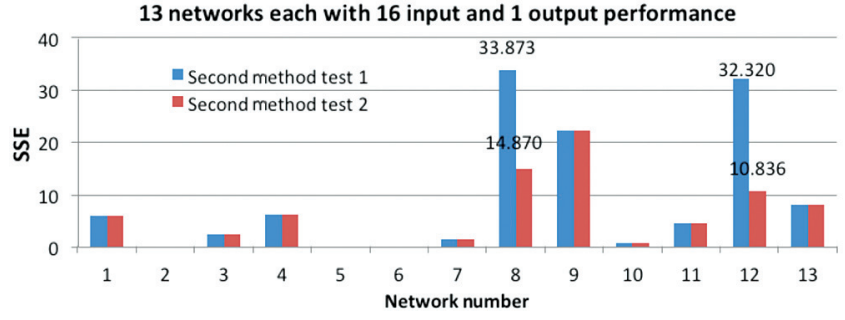

Figure 7. Flexibility of ANN architecture 2 in training stage

loading cases were introduced to it. Having a good performance, the ANN should be able to estimate the external pressure loads that caused those structure responses. For instance, introducing new sets of strain data that have not been used to train the network, the ANN estimates the corresponding load data. Depending on how well the network is trained (the performance of the network), there will be errors between the expected output dataset and the network estimated output (loads). Figure 8 depicts a random example of estimated loads with the ANN for both FEA and experimental tests against the desired loads applied in tests when there was only one external load of $500 \mathrm{~N}$ at location L13 (data is used to train the network). For both sets of problem data it can be seen that the ANN can again estimate the load at the loaded locations with a high

Table 4. Optimum ANN architecture 2

\begin{tabular}{|l|c|}
\hline & 13 networks each with 16 strain inputs and 1 load output \\
\hline Number of networks & 13 \\
\hline Architecture & Feed forward backpropagation \\
\hline Number of layers in each network & Most of it has 1 and for location 8 and 12 are 2 \\
\hline Range of loads & 16 \\
\hline Number of inputs (surface strains) & 1 \\
\hline Number of neurons in output layer (normal loads) & {$[50]$ or [20 20] } \\
\hline Number of neurons in each hidden layer & 1040 \\
\hline Number of training patterns & 1040 \\
\hline Number of testing patterns &
\end{tabular}


degree of accuracy. However, the error size of estimated loads with the ANN for experimental tests is slightly bigger. Such a small error is normal and it could be from an initial error between the FEA data and experimental data, errors induced from the repeatability of the data acquisition system with a resolution of $+/-0.1$ microstrain as well as possible overtraining of the ANN.
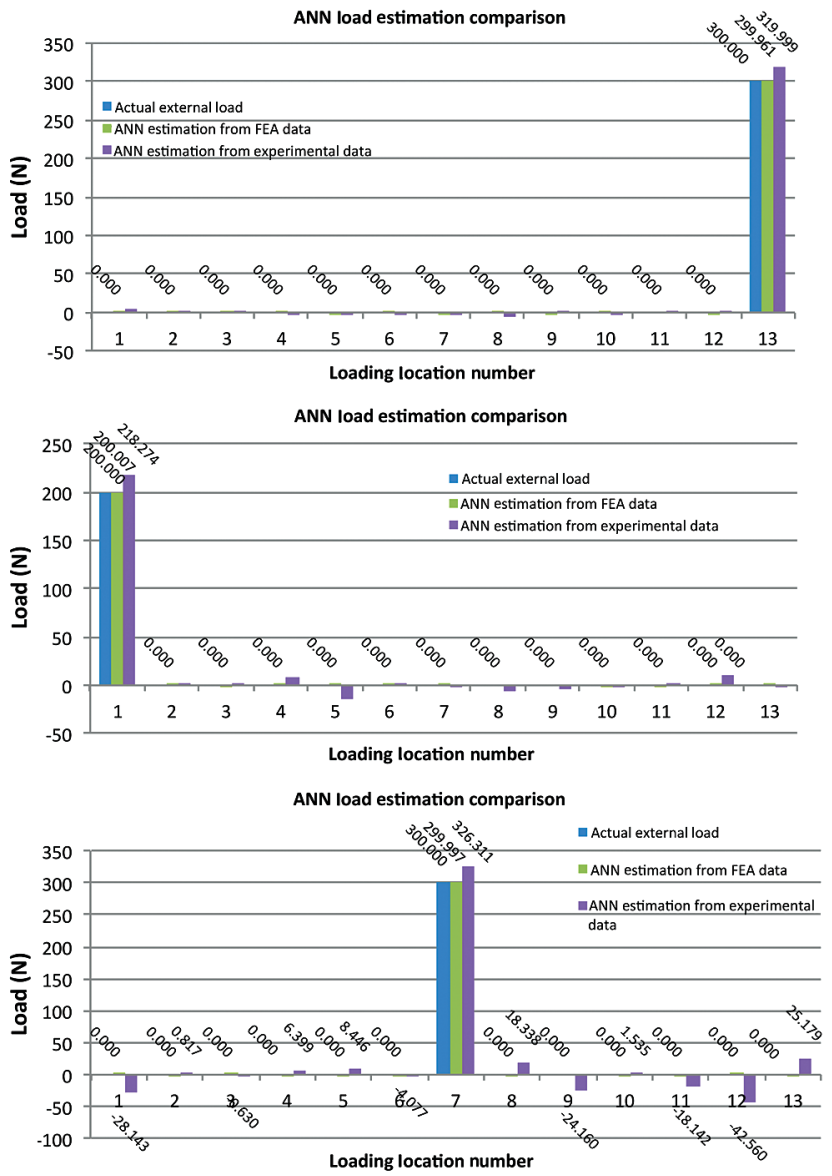

Figure 8. ANN estimation for FEA and experimental data versus expected real data

The estimated negative load values at the unloaded locations were due to the differences between the strain data collected to generate the training data and the collected problem strain data. Due to these errors, slightly different strain patterns are introduced to the ANN producing the errors in the estimated loads. The introduction of further noisy patterns in the training dataset may reduce these small errors, indicating that further work could be carried out to improve the accuracy further.

\section{Discussion}

In this study, it is shown that the inverse problem method, utilising an ANN, is capable of estimating magnitude and position of the static pressure loads on a marine composite panel under large displacement from non-linear strain measurements. The results of this study can be summarised as follows:

$\square$ FEA data can be used to generate training data for ANN inverse load estimation problems.

$\square$ Two different ANN architectures are used and the performances are compared.

$\square$ Having non-linear relationships between the applied load and the surface strains, the system always converges and the SSE is in the range of acceptable error.

The system is capable of estimating the position and magnitude of static pressure loads on a marine composite panel under large displacement. $\square$ Having a large difference between the training datasets and the problem dataset makes the ANN unable to estimate the load accurately.

$\square$ The main source of error was found to be an initial error between the FEA data and experimental data.

The ability to measure the actual load history of a craft in-service would enable the designer to validate the load estimation and structural design tools used during the design stage of a craft. This would lead to the development of more optimal structure designs for this type of craft. The operational safety of the craft can also be improved by having a real-time load monitoring system that is able to detect any degradation of the structural integrity and defects within the structure.

It is proposed that the ANN methodology, with further research and development, could be utilised for the quantification of in-service, transient loads in real-time acting on the craft from the craft's structural response (strain response to load). This would provide valuable information to influence future craft design. In order to fully evaluate the proposed methodology for in-service load monitoring of marine structures, the following areas require investigation:

$\square$ The behaviour of marine structures under transient load conditions (dynamic load is applied).

The effect of the size of the structure on the ANN estimation accuracy.

The number of sensors required for accurate load estimation by optimising the method. While some vessels do have integrated sensors most do not. The number of sensors should be minimised to reduce the time to train the system, cost and weight.

$\square$ The effect of modifying ANN training parameters, including the number and type of training patterns introduced to the ANN.

$\checkmark$ Validation of the methodology on a craft in-service.

Finally, a graphical user interface (GUI) should be developed allowing control of various parameters of the data acquisition and load monitoring system, as well as graphical display in real time.

\section{Conclusions}

It has been shown that the inverse problem approach can be used to estimate the magnitude and position of static pressure loads on a marine composite panel under large displacement from nonlinear strain measurements. A comparison of the ANN loads with the actual applied loads indicated a very good performance of the methodology. This was achieved in real time, providing an accurate load history. This potentially makes the system ideal for solving many classes of complex engineering problem that require load monitoring.

\section{References}

1. W J Pierson, 'A unified mathematical theory for the analysis of propagation and refraction of storm-generated ocean surface waves', part i and ii, New York University, New York, 1952.

2. M St Denis and W J Pierson, 'On the motions of ships in confused seas', SNAME Transactions, Vol 61, 1953.

3. W J Pierson, G Neumann and R W James, 'Practical methods for observing and forecasting ocean waves by means of wave spectra and statistics', Hydrographic Office Publication, 1955.

4. N Hogben, N M C Dacunha and G F Olliver, Global Wave Statistics, Unwin Brothers Ltd, UK, 1986.

5. Y Bai, Marine Structural Design, Elsevier Science Ltd, Oxford, 2003.

6. B V Korvin-Kroukovsky, 'Investigation of ship motions in regular waves', SNAME Transactions, Vol 63, pp 386-435, 1955.

7. J Gerritsma and W Beukelman, 'The distribution of the 
hydrodynamic forces on a heaving and pitching ship model in still water', paper presented at the Fifth Symposium on Naval Hydrodynamics, Washington, 1964.

8. R E D Bishop and W G Price, Hydroelasticity of Ships, Cambridge University Press, Cambridge, 1979.

9. B P Phelps, 'Determination of wave loads for ship structural analysis', DSTO Aeronautical and Maritime Research Laboratory, Melbourne, 1997.

10. J D Clarke, 'Wave loading of warships', Journal of Naval Science, Vol 12, No 4, 1986.

11. T Kukkanen, 'Wave load predictions for marine structures', Rakenteiden Mekaniikka (Journal of Structural Mechanics), Vol 43, No 3, pp 150-166, 2010.

12. H Iwashita, A Ito, T Okada, M Ohkusu, M Takaki and S Mizoguchi, 'Wave force acting on a blunt ship with forward speed in oblique sea', Journal of Society Naval Architecture Japan, Vol 176, 1994.

13. D Nakos and P Sclavounos, 'Ship motions by a threedimensional rankine panel method', paper presented at the Proceedings of 18th Symposium on Naval Hydrodynamics, Ann Arbor, 1990.

14. K Takagi, 'Calculation of unsteady pressure by rankine source method', Journal of Kansai Society of Naval Architecture, Vol 219, pp 47-56, 1993.

15. H Yasukawa, 'A rankine panel method to calculate unsteady ship hydrodynamic forces', Journal of Society Naval Architecture, Vol 168, pp 131-140, 1990.

16. X Cao, Y Sugiyama and Y Mitsui, 'Application of artificial neural networks to load identification', Computers \& Structures, Vol 69, pp 63-67, 1998.

17. R Amali, S Noroozi and J Vinney, 'The application of combined artificial neural network and finite element method in domain problems', paper presented at the Sixth International Conference on Engineering Applications of Neural Networks (EANN 2000), Kingston upon Thames, 2000.

18. R Amali, S Noroozi, J Vinney, P Sewell and S Andrews, 'Predicting interfacial loads between the prosthetic socket and the residual limb for below-knee amputees - a case study', Strain, Vol 42, pp 3-10, 2006.

19. P Sewell, S Noroozi, J Vinney, R Amali and S Andrews, 'Improvements in the accuracy of an inverse problem engine's output for the prediction of below-knee prosthetic socket interfacial loads', Engineering Applications of Artificial Intelligence, Vol 23, pp 1000-1011, 2010.

20. M R Ramazani, S Noroozi, M Koohgilani, B Cripps and P Sewell, 'Determination of static pressure loads on a marine composite panel from strain measurements utilising artificial neural networks', submitted to Proceedings of the Institution of Mechanical Engineers, Part M: J Eng for the Maritime Environment, 1475090212449231, first published on 25 June 2012, doi:10.1177/1475090212449231

21. A P Boresi, R J Schmidt and O M Sidebottom, Advanced Mechanics of Materials, Fifth Edition, John Wiley, 1993.

22. H M Westergaard and W A Salter, 'Moments and stresses in slabs', Proceedings of Amer Concrete Inst, Vol 17, pp 415-538, 1921. 